\title{
Rheology Analysis of Multiphase Flow and its Modeling
}

\author{
Han Zhihong, Liu shuyang \\ Jingdezhen Ceramic Institute, \\ Jingdezhen 333403, China \\ hanliuyang@163.com
}

\begin{abstract}
Most multiphase flow models use viscosity as an important parameter, however, the viscosity of mixed fluid sometimes is unavailable or difficult to measure especially in small-scale flowing condition. In order to solve this problem, a two-phase wedge-sliding model is developed in this paper and this model is non-viscosity dependent. Two variables of Driftinhibition angle and expasion-inhibition angle were defined and their expressions were deduced from the model, which can well index the phase-drift trend of mixed fluid. The study also found that there is an optimal ratio in the ingredients design of mixed fluid and mixed fluid can only be stable when the volume ratio of heavier phase is smaller than this optimal value.
\end{abstract} drift

Keywords - mixed fluid; two-phase wedge-sliding model; phase

\section{INTRODUCTION}

The phase separation and sediment of mixed fluid is a multi-scale and multi-factors coupling process, especially as micro-particle-added lubricant used in the porous material, rheological behaviors present a very complex phenomenon.

Some mltiphase flow models have been successfully proposed by researchers to describe the rheological behaviors in the certain application before, and nearly all these models regard viscosity as an important even key parameter. Early in 1962, Higgins and Leighton [1, 2] presented a fast method to calculate thoroughly the performance of two-phase flow in reservoir rock with complex geometry. By using Stokesian Dynamics, Nott and Brady [3] conducted dynamic simulations of the pressure-driven flow in a channel of a non-Brownian suspension at zero Reynolds number. Ali and Mohamed [4] derived a nonlinear 3D model that investigates the flowdiffusion-structure interaction occurring in mixtures. This is the result of the application of current computer technology and advanced research method in the research field of multi-phase fluids.

But there is no evidence shown that viscosity of mixed fluid is related with the phase separation and sediment at a long-term or stable state; The ingredients separation and sediment of mixed fluid is a long-term accumulated process under stress; In addition, the viscosity of mixed fluid is varying with the temperature, stress, and time, and is difficult to measure even unavailable.

Therefore, when present conventional models are inappropriate to describe the rheological behaviors of mixed fluid accurately, it is necessary to develop a new model with non-viscosity dependence.

\section{FOUNDATION OF MODELING}

A number of balls with same diameter but two different weights are random tiled and formed as a close-packed hexagon-shape which was exactly circumscribed by the inner surface of the barrel without stacking.

According to the theory of mechanics, rotating balls around center had lower kinetic energy, which explained the final distribution of brown (heavier) balls. This result also meets the principle of minimum entropy in the circulation system.

Supposing that all these balls were the structure micro-cell of the mixed fluid, accordingly the heavier phase will have a trend to drift along the stress gradient to lower kinetic energy field. The researches of Cohen [5] on the polymer-phase flow problem, Hatzikiakos [6, 7] and Kalika [8] on the flow of different density polyethylene, and Yoshimura [9] on the suspension of clay and paraffin oil-water emulsion etc., all obtain the similar conclusion that larger molecules phase has trend to drift along the way of stress reduction in the nonuniform stress field, and cause the significantly viscosity decreasing the area of higher stress.

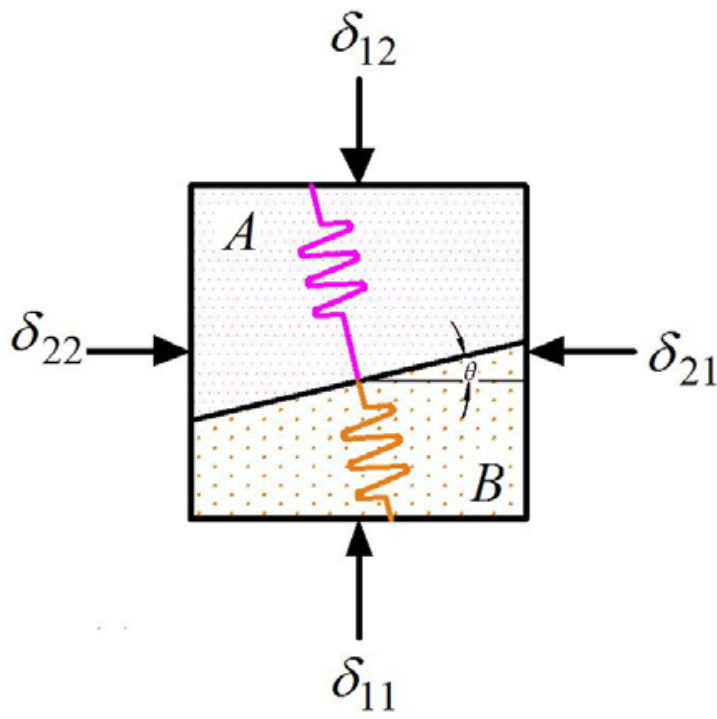

Fig. 1. The unit cell of the two-phase wedge-sliding model 


\section{The Two-Phase WedGe-SLIDING MOdeL}

Assuming that the mixed fluid were a uniform mixture of heavier micro-cell (added phase) A and basic micro-cell (basic phase) B, this well-mixed fluid could be categorized as a typical rheological stability question of two-phase flowing fluid.

Extracting a unit cell from the mixed fluid and assuming the faying surface of two phases in a cell was a wedge, the basic two-phase wedge-sliding model would be generated as shown in Fig. 1.

The action line of the forces is perpendicular to the wedge surface.

where, $\delta_{y}$ is the yield stress of cell $\left(\mathrm{N} / \mathrm{mm}^{2}\right)$, which is related to the environmental temperature and pressure and assumed to be a constant here; $\lambda_{\delta}$ is the prior coefficient of power exponential and defined as,

$$
\lambda_{\delta}=\frac{e^{\left(\rho_{A} \cdot \varphi_{A}+\rho_{B} \cdot \varphi_{B}\right) / 2}}{e^{\rho_{A} \cdot \varphi_{A}}+e^{\rho_{B} \cdot \varphi_{B}}}
$$

Clearly, $0<\lambda_{\delta}<1$ and which is no unit.

The $\lambda_{k}$ is the elastic-plastic anti-force coefficient of the cell.

Obviously, $F_{f}$ is the maximum static friction of two phases when flow reached its steady state, so $F_{f}$ can be defined as:

$$
F_{f}=\lambda_{f} \cdot\left(F_{k A}+F_{k B}\right)
$$

The acting direction of friction is parallel to the wedge surface but opposite with the trend of movement.

The $\lambda_{f}$ is the maximum static friction coefficient between two phases, which is related to the environmental temperature and pressure and assumed to be a constant here.

Defining $\varphi_{B}=\lambda, \lambda \in(0,1)$, then $\varphi_{A}=1-\lambda$. Substituting $\varphi_{A}$ and $\varphi_{B}$ by $\lambda$, the equation above can be rewritten as:

$$
F_{f}=\lambda_{f} \cdot \frac{\rho_{A}{ }^{2} \cdot(1-\lambda)^{2}+\rho_{B}{ }^{2} \cdot \lambda^{2}}{\left.\rho_{A} \cdot(1-\lambda)+\rho_{B} \cdot \lambda\right)} \cdot \frac{e^{\lambda_{\delta} \cdot \delta_{y}}+e^{-\lambda_{\delta} \cdot \delta_{y}}}{e^{\lambda_{\delta} \cdot \delta_{y}}-e^{-\lambda_{\delta} \cdot \delta_{y}}}
$$

Defining:

$$
C_{\rho}=\frac{\rho_{A}{ }^{2} \cdot(1-\lambda)^{2}+\rho_{B}{ }^{2} \cdot \lambda^{2}}{\rho_{A} \cdot(1-\lambda)+\rho_{B} \cdot \lambda}
$$

\section{The STREsSES EQUILIBRIUM ANALYSIS}

Assuming the mixed fluid steadily flows caused by the uniform pressure difference $\Delta P=P_{1}-P_{2}$ between two ends in a slot.

At the parallel direction of wedge surface,

$$
\left(\delta_{12}-\delta_{11}\right) \cdot \sin \theta+\left(\delta_{22}-\delta_{21}\right) \cdot \cos \theta-F_{f}=0
$$

At the perpendicular direction of wedge surface,

$$
\left(\delta_{12}-\delta_{11}\right) \cdot \cos \theta+\left(\delta_{22}-\delta_{21}\right) \cdot \sin \theta-\left(F_{k A}+F_{k B}\right)=0
$$

The longitudinal pressure differential and the transversal expansion-inhibition force are:

$$
\begin{gathered}
\delta=\frac{\Delta p}{A_{p}}=\delta_{12}-\delta_{11} \\
C_{h}=\delta_{21}-\delta_{22}
\end{gathered}
$$
slot.

where, $A_{p}$ is the transversal surface area of the fluid in the

Defining $\theta_{c}=\arctan \left(\frac{C_{h}}{\delta}\right)$, the equation above can be rewritten as:

$$
\sqrt{\delta^{2}+C_{h}^{2}} \cdot \sin \left(\theta-\theta_{c}\right)=\lambda_{f} \cdot \lambda_{k} \cdot C_{\rho} \cdot \frac{e^{\lambda_{\delta} \cdot \delta_{y}}-e^{-\lambda_{\delta} \cdot \delta_{y}}}{e^{\lambda_{\delta} \cdot \delta_{y}}+e^{-\lambda_{\delta} \cdot \delta_{y}}}
$$

Then the wedge angle can be obtained by solving the (9):

$$
\begin{gathered}
\theta=\arcsin \left[\frac{\lambda_{f} \cdot \lambda_{k} \cdot C_{\rho}}{\sqrt{\delta^{2}+C_{h}^{2}}} \cdot \frac{e^{\lambda_{\delta} \cdot \delta_{y}}-e^{-\lambda_{\delta} \cdot \delta_{y}}}{e^{\lambda_{\delta} \cdot \delta_{y}}+e^{-\lambda_{\delta} \cdot \delta_{y}}}\right]+\theta_{C} \\
\theta \in\left[-\frac{\pi}{2},+\frac{\pi}{2}\right]
\end{gathered}
$$

It's clearly that $\theta$ and $\theta_{c}$ respectively indicate the moving trend of phase $\mathrm{B}$ in the longitudinal and transversal directions within cells, so $\theta$ is named as drift-inhibition angle, and $\theta_{c}$ as expansion-inhibition angle here.

Substituting $\lambda_{0}=\Phi_{B}$, then the initial joint stress modulus is gotten:

$$
C_{\rho 0}=\frac{\rho_{A}^{2} \cdot\left(1-\lambda_{0}\right)^{2}+\rho_{B}^{2} \cdot \lambda_{0}^{2}}{\left.\rho_{A} \cdot\left(1-\lambda_{0}\right)+\rho_{B} \cdot \lambda_{0}\right)}
$$

Accordingly, the initial drift-inhibition angle is:

$$
\theta_{0}=\arcsin \left[\frac{\lambda_{f} \cdot \lambda_{k} \cdot C_{\rho 0}}{\sqrt{\delta^{2}+C_{h}^{2}}} \cdot \frac{e^{\lambda_{\delta} \cdot \delta_{y}}-e^{-\lambda_{\delta} \cdot \delta_{y}}}{e^{\lambda_{\delta} \cdot \delta_{y}}+e^{-\lambda_{\delta} \cdot \delta_{y}}}\right]+\theta_{C}
$$




\section{CONCLUSION}

(1) The study shows that these two variables can well index the phase-drift trend of mixed fluid.

(2) An optimal volume ratio of heavier gradient was found and its calculation formula was deduced

\section{REFERENCES}

[1] R.V. Higgins, and A.J. Leighton, "A Computer Method to Calculate Two-Phase Flow in Any Irregularly Bounded Porous Medium”, J. Petrol. Technol. vol. 14, no. 6, pp. 679-683, 1962.

[2] R.V. Higgins, and A.J. Leighton, "Computer Prediction of Water Drive of Oil and Gas Mixtures Through Irregularly Bounded Porous Media Three-Phase Flow”, J. Petrol. Technol. vol. 14, no. 9, pp. 1048-1054, 1962.

[3] R. Nott, Prabhu and F. Brady, John. "Pressure-driven flow of suspensions: simulation and theory”. J. Fluid Mech. vol. 275, pp. 157199, 1994.
[4] A. El Afif, and M. El Omari. "Flow and mass transport in blends of immiscible viscoelastic polymers", Rheol. Acta. vol. 48, no. 3, pp. 285299, 2009.

[5] Y. Cohen, and A.B. Metzner. "Apparent slip flow of polymer solutions”, J. Rheol. vol. 29, no. 1, pp. 67-102, 1985.

[6] S.G. Hatzikiakos, J.M. Dealy, "Wall slip of molten high density polyethylenes. I: Sliding plate rheometer studies”, J. Rheol. vol. 35, no. 4, pp. 497-523, 1991.

[7] S.G. Hatzikiakos, and J.M. Dealy, "Wall slip of molten high density polyethylene. II : Capillary rheometer studies", J. Rheol. vol. 36, no. 4, pp. 703-741, 1992.

[8] D.S. Kalika, M.M. Dean, "Wall slip and extrudate distortion in linear low-density polyethylene”, J. Rheol. vol. 31, no. 8, pp. 815-834, 1987.

[9] A.S. Yoshimura, and R.K. Prudhomme. "Wall slip corrections for couette and parallel disk viscometers”, J. Rheol. vol. 32, no. 1, pp. 53-67, 1985. 